\title{
Heterozygote deficiencies in parasite populations: an evaluation of interrelated hypotheses in the raccoon tick, Ixodes texanus
}

\author{
G Dharmarajan, JC Beasley and OE Rhodes Jr \\ Department of Forestry and Natural Resources, Purdue University, West Lafayette, IN, USA
}

\begin{abstract}
Population genetics is increasingly being used to study the biology of parasites at the scales of both the host (infrapopulation, IP) and host population (component population, $\mathrm{CP})$. In this study we tested three mechanistic hypotheses that could explain deviations from Hardy-Weinberg equilibrium (HWE) expectations due to heterozygote deficits (HDs) at the CP scale in raccoon ticks (Ixodes texanus; $n=718$ ) collected from raccoons (Procyon lotor, $n=91$ ) and genotyped at 11 microsatellite loci. These hypotheses were presence of technical issues (for example, null alleles), hierarchical structure (for example, host demography) and cryptic structure (for example, kin structure). Although statistical support for null alleles existed, their presence would also be expected to lead to an underestimation in levels of relatedness, and thus kin structure. However, we found the opposite pattern:
\end{abstract}

significant HD at the IP scale being more likely in CPs with significant vs non-significant levels of kin structure. Our analyses revealed that pooling of kin groups could lead to highly variable levels of $F_{I S}$ among loci, a pattern usually suggestive of null alleles. We used Monte-Carlo (MC) simulations to show that the existence of subdivided breeding groups and high variance in individual reproductive success could adequately explain deviations from HWE in $I$. texanus. Thus, our results indicate that biological factors can lead to patterns that have usually been interpreted as technical issues (for example, null alleles), and that it is important to take such factors into consideration because loci deviating from HWE likely reflect the effects of real biological processes.

Heredity (2011) 106, 253-260; doi:10.1038/hdy.2010.84; published online 7 July 2010

Keywords: Ixodes texanus; raccoon tick; parasite; microsatellite; component population; Hardy-Weinberg equilibrium

\section{Introduction}

Ecological and microevolutionary processes impacting parasite populations are increasingly being studied using population genetic analyses (reviewed by Criscione et al., 2005; de Meeûs et al., 2007; Criscione, 2008; Archie et al., 2009; for this study the term 'parasite' is restricted to macro-parasites; sensu Lafferty and Kuris, 2002). For example, numerous aspects of a parasite's biology, including mating patterns and transmission dynamics, may be expected to lead to deviations of genotypic frequencies from Hardy-Weinberg equilibrium (HWE) expectations (Criscione et al., 2005; Criscione, 2008). Interestingly, deviations of genotypic frequencies due to heterozygote deficits (HDs) are not uncommon in parasite populations (for example, Plantard and Porte, 2004; Guzinski et al., 2009; Dharmarajan et al., 2010; but also see Caillaud et al., 2006; Keeney et al., 2007; Thiele et al., 2008).

When observed, HDs in parasite populations have historically been attributed to two main causes: the first being technical issues (for example, null alleles; de Meeûs et al., 2004; Roed et al., 2006; Wielgoss et al.,

Correspondence: $\operatorname{Dr} G$ Dharmarajan, Department of Forestry and Natural Resources, Purdue University, Pfendler Building, West Lafayette, IN 47907, USA.

E-mail: gdharmar@purdue.edu

Received 11 October 2009; revised 13 May 2010; accepted 27 May 2010; published online 7 July 2010
2008), and the second being related to sampling issues associated with parasite social systems, movement behavior and/or mating tactics leading to a Wahlund effect (for example, Luo et al., 2003; McCoy et al., 2003; Chevillon et al., 2007; Criscione et al, 2007; Ravel et al., 2007) or inbreeding (for example, Picard et al., 2004; Plantard et al., 2008).

Ticks are major vectors of diseases affecting humans and animals (Jongejan and Uilenberg, 2004) and population genetic tools have the potential to further our understanding of their biology (McCoy, 2008). Although numerous studies have explored the factors affecting genotypic frequencies in parasite populations (see references above), few systematic evaluations of alternative causal hypotheses for deviations from HWE in ticks exist (for example, Chevillon et al., 2007). Our study organism, the raccoon tick (Ixodes texanus), shows a three-host life cycle with all stages (larvae, nymph and adults) predominantly parasitizing the raccoon, Procyon lotor (Darsie and Anastos, 1957). Being a nidicolous tick, I. texanus lives in shelters used by their vertebrate hosts, and in contrast to non-nidicolous ticks, mating in this species usually takes place on the ground rather than on the host (Sonenshine, 1993). In I. texanus deviations from HWE may be caused by three mechanistic factors: technical issues, hierarchical population structure and cryptic structure.

Technical issues are generally associated with problems experienced during polymerase chain reaction 
amplification of genomic DNA (that is, genotyping error due to stutter, large allele dropout and/or null alleles), and can lead to a deficit of heterozygotes compared with HWE expectations. Alternatively, the presence of undetected hierarchical population structure within a sample of ticks can also lead to deviations from HWE because of the inadvertent pooling of individuals from different populations (that is, a Wahlund effect). Such inadvertent pooling of populations is quite likely, because ticks are biologically structured at numerous orthogonal and/or nested levels. For example, ticks are structured with respect to life-history stage (adults and nymphs, in our case), which are further subdivided into infrapopulations (IPs; that is, ticks of a particular lifehistory stage on a particular host individual). In addition, the hosts themselves are heterogeneous with respect to age and sex. The presence of genetic differentiation among any of these nested and/or orthogonal levels could lead to a Wahlund effect (and thus HD) when ticks are pooled at the scale of the component population (CP; that is, ticks of a particular life-history stage infecting a spatially distinct group of hosts).

Finally, the presence of cryptic population structure could also lead to a Wahlund effect when ignored. Numerous cryptic factors could lead to population genetic structure in I. texanus. For example, because I. texanus transmission takes place only at the den site, raccoons that use dens in habitat patches other than the one in which they are trapped may have a genetically differentiated cohort of ticks compared with resident raccoons. Although there is no clear a priori method of differentiating between these two groups of raccoons, pooling ticks from these microgeographic groups at the CP scale would be expected to lead to a Wahlund effect. Alternatively, the presence of kin structure at the $\mathrm{CP}$ scale (for example, the presence of groups of related individuals because of variance in reproductive success of individual ticks) may lead to a 'family' Wahlund effect (Pudovkin et al., 1996).

In this study we genotyped 718 I. texanus adult and nymphal ticks collected from raccoons ( $P$. lotor) at 11 microsatellite loci with the objective of identifying the major mechanism(s) contributing to the HD in this parasite species. To achieve our objective we tested three inter-related hypotheses that could lead to deviations from HWE: the presence of technical issues, hierarchical structure and cryptic population structure.

\section{Materials and methods}

\section{Sample collection and microsatellite data}

I. texanus ticks $(n=736)$ were collected in the spring of 2006 from 91 raccoons trapped in five habitat patches (Pop_01, Pop_03, Pop_14, Pop_16 and Pop_17) located in the Upper Wabash River Basin, Indiana (Supplementary Figure S1; see Table 1 for sampling details; patch numbers follow Dharmarajan et al., 2009a). The patches were separated by distances ranging from 5.98 to $28.06 \mathrm{~km}$, and raccoons inhabiting them were genetically differentiated $\left(0.02 \leqslant \mathrm{~F}_{\mathrm{ST}} \leqslant 0.06 ; P<0.001\right.$ for all pairwise comparisons; Dharmarajan et al., 2009a). Raccoon trapping, immobilization and post-capture processing have been described earlier (Beasley et al., 2007; Beasley and Rhodes, 2008). After raccoons were immobilized, all visible adult/nymphal ticks were collected and stored in 95\% ethanol before DNA extraction. The period of raccoon immobilization was short and thus larval ticks could not be collected, the time required for collecting them being prohibitive.

Ticks were genotyped at 11 microsatellite loci following the protocols reported in Dharmarajan et al. (2009b). We used quality control measures that were standard in our lab (for example, the inclusion of pre-amplified allelic standards in each electrophoretic run and the rescoring of a random subset of samples by an independent scorer; see Drauch et al., 2008) to ensure that the proportions of genotyping error and missing data were $<0.01$ across all loci.

Allelic richness, observed heterozygosity and Nei (1987) unbiased expected heterozygosity were calculated for all ticks collected within each habitat patch using the program GENETIX (Belkhir et al., 2004). As our tick sample consisted of nymphs and adults, all subsequent analyses were carried out separately in these life-history stages. We use the term IP to represent ticks of a particular lifehistory stage infesting a host individual, and the term $\mathrm{CP}$ to represent ticks of a particular life-history stage infecting a spatially distinct group of hosts. Only nine of the possible ten CPs (five patches $\times$ two life-history stages) were used for all subsequent analyses; one CP (adult ticks from Pop_14) was excluded because of low sample size (five individuals; Table 1).

Levels of linkage disequilibrium (LD) for all pairwise locus combinations were tested using the genotypic randomization test (10000 randomizations) implemented

Table 1 Sampling and genetic information for Ixodes texanus ticks collected from five raccoon subpopulations located in Northern Indiana

\begin{tabular}{|c|c|c|c|c|c|c|c|c|c|}
\hline Sub-population & $\begin{array}{c}\text { Hosts } \\
\text { (prop. } \\
\text { infected })^{\mathrm{a}}\end{array}$ & $\begin{array}{c}\text { Ticks } \\
\text { (prop. }^{\text {protyped })^{\mathrm{b}}}\end{array}$ & $\begin{array}{c}\text { Male } \\
\text { yearling } \\
\text { hosts }(\text { ticks) }\end{array}$ & $\begin{array}{c}\text { Male } \\
\text { adult } \\
\text { hosts }(\text { ticks) }\end{array}$ & $\begin{array}{c}\text { Female } \\
\text { yearling } \\
\text { hosts }(\text { ticks) }\end{array}$ & $\begin{array}{c}\text { Female } \\
\text { adult hosts } \\
\text { (ticks) }^{\mathrm{c}}\end{array}$ & $\begin{array}{c}\text { Adult } \\
\text { ticks } \\
\text { (nymphs) }\end{array}$ & $\begin{array}{c}\text { Av. } \\
\text { allelic } \\
\text { richness }\end{array}$ & $H_{\mathrm{S}}\left(H_{\mathrm{O}}\right)$ \\
\hline Pop_01 & $33(0.75)$ & $248(0.95)$ & $12(81)$ & $9(65)$ & $4(66)$ & $8(36)$ & $165(83)$ & 14.63 & $0.795(0.745)$ \\
\hline Pop_03 & $10(0.77)$ & $97(0.98)$ & $3(6)$ & $2(23)$ & $1(29)$ & $4(39)$ & $53(44)$ & 12.91 & $0.802(0.761)$ \\
\hline Pop_14 & $5(0.83)$ & $157(0.99)$ & $1(30)$ & $2(6)$ & $2(121)$ & $0(0)$ & $5(152)$ & 13.27 & $0.791(0.736)$ \\
\hline Pop_16 & $11(0.79)$ & $139(1.00)$ & $2(9)$ & $1(3)$ & $2(56)$ & $6(71)$ & $77(62)$ & 14.09 & $0.797(0.751)$ \\
\hline Pop_17 & $13(0.93)$ & $77(0.97)$ & $5(33)$ & $1(2)$ & $6(29)$ & $1(13)$ & $59(18)$ & 12.91 & $0.782(0.749)$ \\
\hline
\end{tabular}

Sample sizes for all host and tick demographic groups, as well as average allelic richness; Nei (1987) unbiased expected heterozygosity $\left(\mathrm{H}_{\mathrm{S}}\right)$ and observed heterozygosity $\left(\mathrm{H}_{\mathrm{O}}\right)$ across the 11 microsatellites used are reported.

${ }^{a}$ Indicates number of hosts infected with ticks; proportion of hosts (Prop) infected to the total trapped given within brackets.

${ }^{\mathrm{b}}$ Indicates number of ticks genotyped and excludes ticks with missing genotypes at $>2$ loci; proportion of ticks genotyped to total collected given within brackets.

'Based on number of infected hosts and/or number of genotyped ticks. 
in the program GDA (Lewis and Zaykin, 2001). Genotypes rather than alleles were randomized to remove the effect of within-locus disequilibrium (that is, deviation from Hardy-Weinberg equilibrium) on pairwise LD levels. We carried out a total of 495 pairwise comparisons (55 locus pairs evaluated in each of 9 CPs). The existence of significant levels of LD across all combinations was tested by calculating the exact binomial probability ( $P_{\text {binomial; }}$ Rosner, 2006) of obtaining the observed number of statistically significant tests $\left(n_{\text {sig }}\right)$ given the number of tests carried out $\left(n_{\text {tests }}\right)$ and the Type I error rate $(\alpha=0.05)$.

We tested for HD at the scales of the IP (within each $\mathrm{CP}), \mathrm{CP}$, and globally using Weir and Cockerham (1984) estimator of $F_{I S}(f)$. The $U$-test was used to test for significant levels of HD using the Markov chain algorithm implemented in GENEPOP007 (dememorization $1 \times 10^{4}$, batches 500, iterations per batch $1 \times 10^{6}$; Rousset, 2008).

To determine whether there was a direct effect of host age and sex on $f$ at the IP scale, we used a generalized linear model with normally distributed errors and an identity link (SPSS version 16; SPSS, Chicago, IL, USA). The initial model was of the form $f \sim$ Site + Host sex + Host age + Host sex:Host age + Constant (where site was a nominal factor with d.f. $=4$, and ' $:$ ' implies an interaction effect). Model parsimony was assessed using Akaike's Information criterion corrected for sample size $\left(\mathrm{AIC}_{\mathrm{C}}\right.$; Burnham and Anderson, 2002).

\section{Technical issues}

We tested for the presence of three technical issues associated with polymerase chain reaction amplification: genotyping error caused by stuttering, large allele dropout and null alleles. We tested for genotyping error caused by stuttering and large allele dropout at the scale of each $\mathrm{CP}$ using the randomization routines (5000 randomizations) implemented in the program MICROCHECKER (Van Oosterhout et al., 2004). In addition, we tested for a signature of large allele dropout at the scales of both the IP and CP by regressing allele-specific

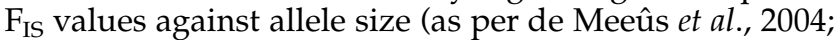
for detailed methodology, see Supplementary Methods).

We initially inferred the presence of null alleles in all locus/IP combinations that revealed significant HD as indicated by the $U$-test (see above). As null homozygotes are expected to appear as missing genotypes (that is, blanks) we also tested for a significant positive relationship between $\mathrm{F}_{\mathrm{IS}}$ at the $j$ th locus in the $i$ th IP $\left(f_{i j}\right)$ and the observed number of blanks using a generalized linear model. The generalized linear model analysis was carried out using a binomial error distribution and a logit link function (SPSS version 16), and the dependent variable was modeled as a binomial count variable (the number of 'successes' (that is, blanks) given the number of trials (that is, individuals genotyped)). We carried out the generalized linear model analysis separately at each locus and across all loci. We also repeated the above analyses to infer the presence of null alleles at the CP scale.

\section{Hierarchical structure}

Heterozygote deficits may occur if non-interbreeding populations are sampled as a single entity, even if each population is independently in HWE (Wahlund, 1928). We tested for the presence of hierarchical genetic structure in each $\mathrm{CP}$ at two nested scales (IP and individual). As genetic structuring of parasite populations could be affected by demographic factors related to the host (age/sex), we controlled for these factors using the method described by de Meeûs and Goudet (2007). We divided ticks within each CP into two groups based on the age of the host (adult vs yearling) and hierarchal analyses were undertaken separately for each group (within each CP). We used the program HIERFSTAT (Goudet, 2005) to estimate genetic variance partitioned among host sexes within host age $\left(\mathrm{F}_{\text {Sex-Age }}\right)$, among IPs within host sex $\left(\mathrm{F}_{\mathrm{IP}-\mathrm{Sex}}\right)$, among individuals within IPs $\left(\mathrm{F}_{\mathrm{I}-\mathrm{IP}}\right)$, among individuals within host sex $\left(\mathrm{F}_{\mathrm{I}-\mathrm{Sex}}\right)$ and among individuals within host age $\left(\mathrm{F}_{\mathrm{I}-\text { Age }}\right)$. We generated $95 \%$ confidence intervals for all the F-statistics by bootstrapping (5000 times) across loci (as implemented in HIERFSTAT).

In the case of $\mathrm{F}_{\mathrm{Sex}-\mathrm{Age}}$ and $\mathrm{F}_{\mathrm{IP}-\mathrm{Sex}}$, we also tested for significance using 5000 randomizations of IPs among host sex and individuals among IPs (within host sex), respectively. Independent $P$-values were combined across CPs using the exact binomial test (Rosner, 2006) in the case of $\mathrm{F}_{\mathrm{I}-\mathrm{IP}}, \mathrm{F}_{\mathrm{I}-\mathrm{Sex}}$ and $\mathrm{F}_{\mathrm{I}-\mathrm{Age}}$ (exact $P$ values being unavailable) and Fisher's method (Sokal and Rohlf, 1981) in the case of $\mathrm{F}_{\text {Sex-Age }}$ and $\mathrm{F}_{\mathrm{IP}-\mathrm{Sex}}$.

In the absence of host age/sex effects, we estimated the genetic variance partitioned among all IPs within each $\mathrm{CP}\left(\mathrm{F}_{\mathrm{IP}-\mathrm{CP}}\right)$ and among $\mathrm{CPs}\left(\mathrm{F}_{\mathrm{CP}-\mathrm{Total}}\right)$, and evaluated the statistical significance by permuting (5000 times) individuals among IPs within CPs (in case of $\mathrm{F}_{\mathrm{IP}-\mathrm{CP}}$ ) and IPs among CPs (in case of $\mathrm{F}_{\mathrm{CP}-\mathrm{Total}}$ ).

\section{Cryptic structure}

As mentioned earlier, cryptic genetic structure could be caused by the presence of microgeographic and family (kin) structure. We tested for the presence of cryptic microgeographic structure using the program BAPS version 5.3 (Corander et al., 2008), which identifies cryptic sub-structure by minimizing HWE and LD within each of $k$ clusters. We used BAPS to estimate $k$ within each CP using 15 runs wherein maximum $k$ was constrained at the number of individuals genotyped. The $k$ having the highest posterior probability (across the 15 runs) was considered the most likely number of clusters identified by BAPS. As BAPS may overestimate $k$ due to the inference of a few small spurious clusters (Latch et al., 2006), we verified the results of the BAPS analyses using the program STRUCTURE version 2.2 (Pritchard et al., 2000; see Supplementary Methods for details of STRUCTURE analyses).

To test for the presence of kin structure at the CP scale, we partitioned individuals into putative kin groups using the program PEDIGREE (Herbinger, 2005). PEDIGREE uses an MCMC algorithm to sample the space of possible partitions of individuals, and calculates for each partition a score based on the pairwise score method described by Smith et al. (2001) and Butler et al. (2004). In order to identify the best partition of individuals into putative kin groups, we performed five PEDIGREE runs for each CP (500000 iterations per run) with the following parameters: 'full-sib constraint' $=0$; 'temperature' $=10$; and 'weight' $=1$ (the functions of each of these three 
parameters are detailed in Supplementary Table S1). The statistical strength of the best partition was tested by performing hundred PEDIGREE runs (using parameters above) after randomizing alleles among individuals (as per Herbinger, 2005), and statistical significance was calculated as the proportion of randomizations producing a partition score $\geqslant$ the observed score.

Although other clustering software (for example, BAPS) have been used to identify the presence of kin structure in ticks (see Chevillon et al., 2007), our empirical evaluation of three algorithms indicated that both BAPS and STRUCTURE have higher $\alpha$ error rates $(\approx 0.20$ and $\approx 0.50$, respectively) compared with PEDIGREE $(\approx 0.03)$ when identifying half-sib groups (Supplementary Figure S2; See Supplementary Methods for the detailed methodology used to calculate error rates). However, PEDIGREE has low power (0.50); thus, estimates of kin structure by PEDIGREE are likely to be conservative (Supplementary Figure S2).

\section{Results}

We successfully genotyped 718 of 736 (98\%) I. texanus ticks at 11 microsatellite loci (Table 1). Ticks sampled from each of the five habitat patches showed high levels of polymorphism (range: 12.9-14.6 alleles/locus) and unbiased expected heterozygosities (range: 0.78-8.0; Table 1). Statistically significant levels of LD were detected in about 5\% (25 of 495) of the locus pairs tested across the nine CPs, which was within binomial expectations given the Type 1 error rate $\left(n_{\text {tests }}=495\right.$; $\left.n_{\text {sig }}=25 ; P_{\text {binomial }}=0.508\right)$. A large number of locus $/ \mathrm{CP}$ combinations $(28 \%)$ showed significant deviations from HWE $\quad\left(n_{\text {tests }}=99 ; \quad n_{\text {sig }}=28 ; \quad P_{\text {binomial }}<0.001 ;\right.$ Table 2$)$. Significant levels of HD were detected in 8 of 9 CPs (across all loci; Table 2) and 6 of 11 loci (across all CPs; Table 2), and the global $F_{I S}$ across all loci and CPs was $0.060(P<0.001)$. The estimates of $F_{\text {IS }}$ calculated at the scale of the CP and IP were highly variable across loci
(Supplementary Figure S3). We found no evidence that this variation was due to the effects of host sex or age; none of the variables were significant in the linear regression, and the intercept-only model was the most parsimonious.

\section{Technical issues}

We found statistical evidence for genotyping error due to stutter in two locus/CP combinations (see Table 2 and Supplementary Figure S4), which was within binomial expectations given the Type I error rate $\left(n_{\text {tests }}=99\right.$; $\left.n_{\text {sig }}=2 ; P_{\text {binomial }}=0.961\right)$. There was no evidence of large allele dropout at any locus, at the scale of the IP or CP (see Supplementary Table S2). We found that 28 of 99 locus/CP combinations examined showed significant levels of null alleles as detected by the $U$-test (Table 2). However, there was no evidence of a significant positive relationship between number of blanks and $\mathrm{F}_{\mathrm{IS}}$ at any locus or across all loci at the scale of the IP or CP (Supplementary Table S3).

\section{Hierarchical structure}

We found no evidence for genetic differentiation among ticks infecting male vs female raccoons or among IPs within each host sex category (for details, see Supplementary Table S4). We also found no significant differences between $\mathrm{F}_{\mathrm{Sex} \text {-Age }}$ or $\mathrm{F}_{\mathrm{IP}-\mathrm{Sex}}$ values between yearling and adult raccoons, as indicated by a complete overlap in bootstrap CIs (see Supplementary Table S4). As raccoon age and sex did significantly affect the genetic structuring of I. texanus, we pooled IPs across host sex and age categories before global analyses. The results of the global analysis revealed that there was no genetic differentiation between IPs within the CPs (adult ticks: $\mathrm{F}_{\mathrm{IP}-\mathrm{CP}} \approx 0, P=0.526$; nymphs: $\mathrm{F}_{\mathrm{IP}-\mathrm{CP}} \approx 0, P=0.413$ ). However, we found low but statistically significant levels of genetic differentiation among the CPs (adult ticks: $\mathrm{F}_{\mathrm{CP}-\text { Total }}=0.002, \quad P<0.001$; nymphs: $\mathrm{F}_{\mathrm{CP}-\text { Total }}=0.005$,

Table 2 Weir and Cockerham's (1984) estimates of $\mathrm{F}_{\mathrm{IS}}(f)$ across nine Ixodes texanus component populations (CP) at 11 loci

\begin{tabular}{|c|c|c|c|c|c|c|c|c|c|c|c|c|c|}
\hline$C P$ & Stat. & IT_05 & IT_06 & IT_10 & $I T \_11$ & $I T \_15$ & IT_19 & $I T \_24$ & IT_26 & $I T \_28$ & IT_30 & IT_34 & All \\
\hline \multirow[t]{2}{*}{ Pop_01 Adult } & $f$ & 0.317 & 0.099 & 0.031 & 0.050 & -0.099 & 0.089 & 0.131 & -0.026 & 0.000 & 0.022 & 0.044 & 0.060 \\
\hline & $P$ & 0.001 & 0.001 & 0.395 & 0.002 & 0.968 & 0.004 & $<0.001$ & 0.578 & 0.509 & 0.276 & 0.434 & 0.001 \\
\hline \multirow{2}{*}{ Pop_01 Nymph } & $f$ & 0.346 & 0.006 & 0.013 & 0.075 & -0.016 & 0.172 & 0.103 & 0.086 & -0.025 & 0.039 & -0.043 & 0.067 \\
\hline & $P$ & $<0.001$ & 0.316 & 0.218 & 0.075 & 0.669 & 0.001 & 0.020 & 0.066 & 0.893 & 0.009 & 0.931 & 0.001 \\
\hline \multirow[t]{2}{*}{ Pop_03 Adult } & $f$ & 0.089 & 0.099 & 0.032 & -0.019 & 0.043 & 0.127 & 0.122 & -0.052 & 0.047 & 0.119 & -0.042 & 0.050 \\
\hline & $P$ & 0.099 & 0.155 & 0.422 & 0.668 & 0.512 & 0.003 & 0.015 & 0.503 & 0.303 & 0.001 & 0.807 & 0.006 \\
\hline \multirow[t]{2}{*}{ Pop_03 Nymph } & $f$ & -0.016 & 0.180 & -0.032 & 0.030 & 0.127 & 0.102 & 0.117 & -0.077 & 0.078 & 0.036 & 0.060 & 0.052 \\
\hline & $P$ & 0.463 & 0.007 & 0.563 & 0.600 & 0.227 & 0.173 & 0.025 & 0.913 & 0.133 & 0.382 & 0. & 0.012 \\
\hline \multirow[t]{2}{*}{ Pop_14 Nymph } & $f$ & $\underline{0.311}$ & 0.079 & 0.063 & -0.012 & 0.005 & 0.136 & 0.238 & 0.044 & 0.007 & -0.024 & 0. & 0.074 \\
\hline & $P$ & $\overline{0.001}$ & 0.119 & 0.389 & 0.606 & 0.494 & 0.001 & $<0.001$ & 0.011 & 0.617 & 0.908 & 0.256 & 0.001 \\
\hline \multirow{2}{*}{ Pop_16 Adult } & $f$ & $\overline{0.215}$ & 0.097 & 0.140 & 0.126 & -0.082 & 0.188 & 0.095 & 0.013 & -0.003 & -0.011 & -0.047 & 0.067 \\
\hline & $P$ & 0.003 & 0.144 & $\underline{0.126}$ & 0.009 & 0.920 & 0.029 & 0.172 & 0.483 & 0.220 & 0.227 & 0.498 & 0.002 \\
\hline \multirow[t]{2}{*}{ Pop_16 Nymph } & $f$ & 0.193 & 0.034 & $-\overline{0.006}$ & 0.181 & 0.115 & -0.053 & 0.086 & -0.015 & 0.017 & -0.018 & 0.025 & 0.048 \\
\hline & $P$ & 0.002 & 0.179 & 0.066 & $<0.001$ & 0.295 & 0.641 & 0.2 & 0.751 & 0.145 & 0.267 & 0.799 & 0.001 \\
\hline \multirow[t]{2}{*}{ Pop_17 Adult } & $f$ & 0.154 & 0.232 & 0.100 & -0.025 & -0.055 & 0.017 & 0.066 & 0.041 & 0.097 & 0.003 & 0.001 & 0.059 \\
\hline & $P$ & 0.007 & $<0.001$ & 0.260 & 0.778 & 0.762 & 0.187 & 0.008 & 0.593 & 0.011 & 0.750 & 0.757 & 0.001 \\
\hline \multirow[t]{2}{*}{ Pop_17 Nymph } & $f$ & 0.145 & 0.092 & -0.053 & 0.009 & -0.222 & -0.014 & -0.063 & -0.023 & 0.021 & 0.020 & -0.160 & -0.014 \\
\hline & $P$ & 0.150 & 0.101 & 0.809 & 0.169 & 0.970 & 0.201 & 0.551 & 0.358 & 0.453 & 0.379 & 0.981 & 0.218 \\
\hline \multirow[t]{2}{*}{ All } & f & 0.242 & 0.094 & 0.043 & 0.046 & -0.020 & 0.103 & 0.131 & 0.008 & 0.017 & 0.014 & 0.005 & 0.060 \\
\hline & $P$ & 0.001 & 0.001 & 0.231 & 0.001 & 0.938 & 0.001 & 0.001 & 0.089 & 0.075 & 0.018 & 0.567 & 0.001 \\
\hline
\end{tabular}

Locus component population (CP) combinations showing significant signature of genotyping error because of stutter are underlined. Deviations from Hardy-Weinberg equilibrium expectations in the direction of heterozygote deficiency were tested using the $U$-test $(P)$ and significant $\mathrm{F}_{\mathrm{IS}}$ values are bolded $(P \leqslant 0.05)$. 
$P<0.001)$. The hierarchical analyses revealed that the maximum genetic variance was usually partitioned at the lowest scale at which we sampled (that is, within individuals at the IP scale; see Supplementary Table S4).

\section{Cryptic structure}

The clustering algorithm used by BAPS identified a variable number of clusters (mean: 7.2; range: 3-11) in every $\mathrm{CP}$ examined; however, in 8 of $9 \mathrm{CPs}$, more than $80 \%$ of individuals were grouped into a single large cluster (see Supplementary Table S5). In contrast to the results of BAPS, analyses with STRUCTURE revealed no evidence of cryptic genetic structure in 8 of 9 CPs (see Supplementary Table S5). The exception to this pattern was nymphs in Pop_03, wherein STRUCTURE identified the presence of two groups of ticks; however, the partition showed little biological relevance because individuals were not strongly assigned to either of the clusters (see Supplementary Table S5).

Our use of the program PEDIGREE revealed significant levels of kin structure in 5 of 9 CPs examined $\left(\chi^{2} 18\right]=52.69 ;$ combined $P$-value $<0.001$; Table 3); however, the kin groups were usually small $(\approx 2.1$ individuals) and regularly pooled individual ticks from different IPs within each CP ( $<5 \%$ individuals within kin groups being from a single host). Interestingly, the probability of an IP showing significant HD was higher than binomial expectations in the majority of CPs showing significant levels of kin structure (that is, 4 of 5 CPs; Table 3), but not in those with non-significant levels of kin structure (that is, 1 of 4 ; Table 3 ). Thus, deviations from HWE expectations at the IP scale were more likely in CPs that exhibited significant vs nonsignificant levels of kin structure.

\section{Post hoc analyses}

Our analyses indicated that the signature of HD originated at the lowest scale at which analyses were performed: the IP. Although the presence of null alleles might be considered to be a simple explanation for the observed HD, this hypothesis received statistical support only with the $U$-test, and thus may be confounded by factors related to parasite biology, such as kin structure (see Discussion). We evaluated whether biological factors (that is, the levels of kin structure and life-history characteristics of $I$. texanus) could adequately explain deviations from HWE at the IP scale using a MC simulation approach. The MC simulation was principally based on the subdivided breeding group model proposed by Criscione and Blouin (2005), modified to take into account the levels of kin structure observed in I. texanus.

In each $\mathrm{CP}$ we carried out the MC simulation in three stages. First, we generated half-sib groups whose sizes reflected the sizes of kin groups identified by PEDIGREE (in CPs with non-significant levels of kin structure we considered all the ticks to be unrelated; that is, the number of kin groups was equal to the number of ticks sampled). The half-sib groups were generated from the allele frequency distribution of an IP selected at random (with replacement). In the second stage we generated a random set of hosts (the number of hosts being same as that in the observed $\mathrm{CP}$ ) and redistributed the kin groups among these hosts to reflect the distribution of kin groups among IPs in the observed data. Finally, we calculated the $\mathrm{F}_{\mathrm{IS}}$ at each locus and across all loci for each randomly generated IP. We repeated the simulation procedure 1000 times to obtain a distribution of $F_{I S}$ values (at each locus and across all loci) for each observed IP. For each IP (at each locus and across all loci) we tested whether the MC simulation provided adequate support for the observed data by calculating the probability of obtaining a simulated value $\geqslant$ observed. We evaluated the overall fit of the model expectations to observed values (at each locus and across all loci) using the exact binomial test described earlier. To visualize the fit of the simulated data to the observed data, we also generated 95\% CIs for each IP (at each locus and across all loci). Although our MC simulation

Table 3 Results of kin group clustering and Monte-Carlo simulation analyses in nine Ixodes texanus component populations (CP)

\begin{tabular}{|c|c|c|c|c|c|}
\hline \multirow[t]{2}{*}{$C P(\mathrm{~N}$ individuals $)$} & \multicolumn{3}{|c|}{ Pedigree analyses } & \multicolumn{2}{|c|}{ Binomial probability $(\mathrm{r} ; \mathrm{n})^{\mathrm{b}}$} \\
\hline & $\mathrm{N}$ groups & Mean $( \pm \sigma)^{\mathrm{b}}$ & Score $(\mathrm{P})^{\mathrm{a}}$ & Panmixia & $M C$ \\
\hline Pop_01 Adult (164) & 54 & $2.352(0.346)$ & $1.75 \times 10^{5}(0.02)$ & $<0.001(7 ; 21)$ & $1.000(0 ; 21)$ \\
\hline Pop_ 01 Nymph (84) & 28 & $2.071(0.069)$ & $4.57 \times 10^{4}(0.04)$ & $<0.001(6 ; 12)$ & $1.000(0 ; 12)$ \\
\hline Pop_03 Adult (53) & 12 & $2.000(0.000)$ & $1.86 \times 10^{4}(0.64)$ & $0.057(2 ; 8)$ & $1.000(0 ; 8)$ \\
\hline Pop_03 Nymph (44) & 8 & $2.000(0.000)$ & $1.30 \times 10^{4}(0.06)$ & $0.143(1 ; 3)$ & $1.000(0 ; 3)$ \\
\hline Pop_14 Nymph (152) & 53 & $2.245(0.227)$ & $1.50 \times 10^{5}(0.03)$ & $<0.001(3 ; 4)$ & $1.000(0 ; 4)$ \\
\hline Pop_ 16 Adult (77) & 19 & $2.316(0.339)$ & $3.88 \times 10^{4}(0.01)$ & $0.008(3 ; 9)$ & $1.000(0 ; 9)$ \\
\hline Pop_16 Nymph (62) & 17 & $2.059(0.059)$ & $2.50 \times 10^{4}(0.14)$ & $0.004(3 ; 7)$ & $1.000(0 ; 7)$ \\
\hline Pop_17 Adult (59) & 17 & $2.118(0.110)$ & $2.26 \times 10^{4}(0.01)$ & $0.057(2 ; 8)$ & $1.000(0 ; 8)$ \\
\hline Pop_ 17 Nymph (18) & 1 & $2.000(-)$ & $2.10 \times 10^{3}(0.28)$ & $1.000(0 ; 5)$ & $1.000(0 ; 5)$ \\
\hline Overall & 23.22 & $2.130(-)$ & $<0.001^{\mathrm{c}}$ & $<0.001(27 ; 77)$ & $1.000(0 ; 77)$ \\
\hline
\end{tabular}

For kin group clustering (PEDIGREE analyses), reported are the number of individuals in the CP ( $N$ ), number of kin groups identified ( $N$ groups), mean ( \pm variance) of kin group sizes, PEDIGREE partition score (score), significance value $(P)$. In addition, exact binomial probabilities to test for heterozygote deficits (HDs) at the scale of the infrapopulation (IP) also are reported. Each IP was evaluated for significant levels of HD as compared with panmictic expectation (Panmixia) and the kin-structured Monte-Carlo simulation (MC). Values that deviate significantly $(\alpha=0.05)$ are in bold.

a Statistical significance based on 100 randomizations; $P$-values indicate the proportion of randomizations in which the partition score $\geqslant$ observed.

${ }^{\mathrm{b}}$ Calculated based number of 'successes' ( $r$; that is, the number of IPs showing significant heterozygote deficits across all loci based on expectations of the specific model, that is, panmictic vs kin-structured) given the number of 'trials' ( $n$; that is, the number of IPs evaluated). 'Independent $P$-values combined using Fisher's method (Sokal and Rohlf, 1981). 
analyses were based on some simplifying assumptions regarding $I$. texanus biology, these assumptions were unlikely to seriously affect our results (see Testing MC simulation assumptions in Supplementary Methods; see also Supplementary Figure S5 and S6).

Across all IPs evaluated we found that the MC simulation adequately explained the observed levels of HD both across all loci (Figure 1; see also Supplementary Table S6) and at each locus (no individual locus deviating from MC expectation across all IPs; Supplementary Table S6 and Figure S7). In addition, at the scale of the CP we found that the number of IPs showing significant levels of HD across all loci (compared with MC simulation expectation) did not deviate from the binomial expectation in any of the nine CPs examined (Table 3 ).

\section{Discussion}

This study evaluated three interrelated hypotheses that may cause genotypic frequencies in I. texanus CPs to deviate from HWE expectations: the existence of technical issues (genotyping error due to stutter, large allele dropout and null alleles), hierarchical structure and cryptic population structure.

The only technical issue for which we obtained statistical support was the presence of null alleles and this hypothesis was only supported by the $U$-test (Rousset, 2008). However, the U-test could lead to the spurious identification of null alleles in the presence of any demographic process (for example, kin structure) that causes genotypic frequencies to deviate from HWE (Dakin and Avise, 2004). We thus return to the null allele hypothesis later (see below).

The second hypothesis that we evaluated as a cause of $\mathrm{HD}$ in $I$. texanus CPs was the evidence of underlying hierarchical genetic structure caused by the interaction between host demography (that is, host age and sex) and the spatial subdivision of ticks into IPs. Given the life cycle of the parasite, we expected that HD (at the $\mathrm{CP}$ scale) could have been caused by the limited movement

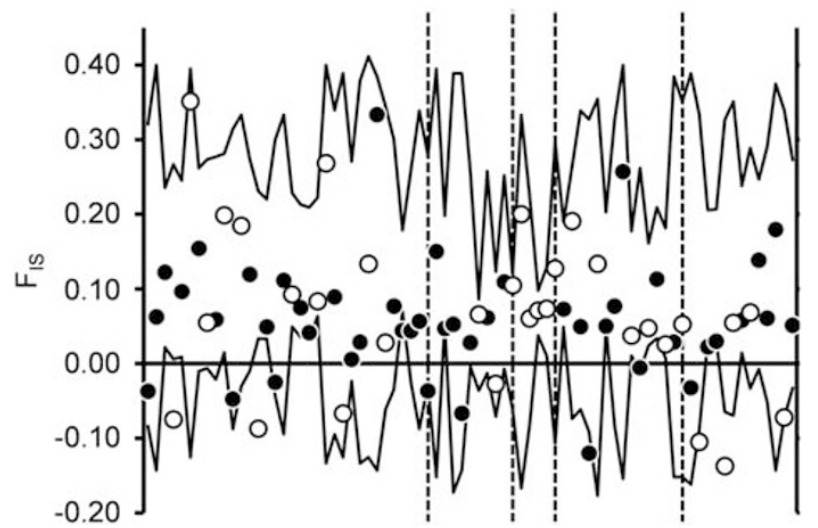

Figure 1 Distribution of observed $F_{\text {IS }}$ values calculated across 11 microsatellites at the scale of the infrapopulation (IP) in the case of adult (closed circles) and nymphal (open circles) Ixodes texanus ticks. The IPs are grouped by the raccoon subpopulations, and discrete subpopulations delineated by dashed lines; the order of subpopulations represented is Pop_01, Pop_03, Pop_14, Pop_16 and Pop_17, respectively. The $95 \%$ confidence intervals based on the kin-structured Monte-Carlo simulation (black lines) are also represented. of ticks among raccoon den sites. Surprisingly, in both adult and nymphal ticks we found very low levels of genetic structure both at the scale of the CP (beacuse of host demography or spatial subdivision) and at the scale of the entire population (that is, among CPs).

The third hypothesis we evaluated was the presence of cryptic microgeographic and/or kin structure. Although our analyses provided no support for the presence of cryptic microgeographic structure, they provided strong statistical support for the presence of kin structure in a large proportion of CPs examined. Empirical research has shown that ignoring social structure can lead to serious biases in inferences drawn from molecular data with regard to the biology of the species being studied (for example, Boone and Rhodes, 1996; Latch and Rhodes, 2006), and this is because cryptic social structure in a population may lead to deviations from HWE (see Sugg et al., 1996). Although our analyses provided strong statistical support for the existence of kin structure in the I. texanus CPs, we note that the microsatellites used have low power in terms of differentiating half-sibs from unrelated individuals (Type II error $\approx 50 \%$ ), making our estimates of kin structure conservative. Although the existence of temporally stable social structure is unlikely in the case of parasites, even transient kin structure because of the presence of admixed kin groups at the CP scale could lead to a HD through a 'family' Wahlund effect (Pudovkin et al., 1996). The presence of admixed sib groups could be a direct result of high variance in female reproductive success (Criscione and Blouin, 2005; Chevillon et al., 2007). Indeed, Chevillon et al. (2007) proposed that the admixture of ticks from distinct sib-groups could have led to significant levels of HD found at the CP scale in the cattle tick (Rhipicephalus microplus).

In I. texanus the presence of a large number of kin groups in a majority of CPs, as well as the high variance in kin group size, lends support to the hypothesis of variance in the reproductive success of individual ticks. However, less than $5 \%$ of the identified kin groups consisted of ticks sampled from a single host, revealing that though there is kin structure within CPs, there are also high levels of gene flow among raccoon dens (a fact supported by the low levels of $\mathrm{F}_{\mathrm{ST}}$ among IPs).

Of the hypotheses explored, only two received statistical support: null alleles and kin structure. Clearly, the high variance in $\mathrm{F}_{\mathrm{IS}}$ across loci at the scale of the $\mathrm{CP}$ and IP is suggestive of the presence of null alleles. On the other hand, three lines of evidence suggest that null alleles were unlikely to be the main cause of deviation from HWE: (1) levels of missing data did not support the null allele hypothesis at the IP or CP scales; (2) our analyses provided strong statistical support for the presence of kin structure in a majority of CPs examined, and analysis of genetic patterns in randomly generated kin-structured populations revealed that pooling kin groups could lead to a pattern usually considered to be a signature of null alleles: high levels of $F_{I S}$ as well as high variance in $\mathrm{F}_{\mathrm{IS}}$ among loci (compare Supplementary Figures S2A and S3); and (3) finally, null alleles are expected to lead to an underestimation of relatedness between individuals (Dakin and Avise, 2004; Wagner et al., 2006); thus, we would expect low levels of kin structure when null allele frequency is high. However, our data show the opposite pattern with evidence that 
$\mathrm{HD}$ at the IP scale was significantly higher in CPs having significant levels of kin structure, compared with those with non-significant levels of kin structure. Given the three reasons mentioned above, it seems unlikely that $\mathrm{HD}$ in I. texanus is caused by the presence of null alleles.

Our post-hoc analyses revealed interesting insights into the biology of $I$. texanus in particular and potentially nidicolous parasites in general. We found that kin structure, when combined with subdivided breeding groups (that is, den sites), has the potential to produce deviation from HWE at the IP scale along with high variance in $\mathrm{F}_{\mathrm{IS}}$ at individual loci. There is no doubt that the model makes certain simplifying assumptions regarding the life-history characteristics of I. texanus, one important assumption being that allele frequencies observed at the IP scale adequately reflected the allele frequencies of parents forming the subsequent generation of ticks. Unfortunately, empirical support for this assumption requires sampling of the dens, which our sampling scheme did not incorporate. However, relatedness and $\mathrm{F}_{\mathrm{IS}}$ values within the MC-simulated kin groups emulated closely those of the observed kin groups, indicating that our assumptions are unlikely to seriously affect the simulation results. It is clear that future studies will need to concentrate on attempting to integrate population genetic patterns at the scale of the dens with those at the scale of the IP.

To summarize, our analyses revealed that in parasite systems showing heterozygote deficiency at the IP scale, the existence of subdivided breeding groups (Criscione and Blouin, 2005) and the high variance in individual reproductive success can lead to deviations from HWE. Importantly, our analyses have revealed that in such systems there is likely to be high variance in $F_{\text {IS }}$ among loci due to random factors affecting the genetic composition of parents contributing progeny as well as a Wahlund effect when these progeny are pooled at the IP scale. Although we do not imply that null alleles could not be a factor leading to HD in either I. texanus or parasites in general, we contend that biological factors can also lead to patterns that have usually been interpreted as being caused by technical issues (for example, null alleles). Our results indicate that it is important to take such biological factors into consideration when addressing HD in natural systems, particularly because loci that deviate from HWE are likely to reflect the effects of real biological processes, and eliminating such loci may be equivalent to 'throwing the baby out with the bath water'.

\section{Conflict of interest}

The authors declare no conflict of interest.

\section{Acknowledgements}

We thank Joseph Busch, Jennifer Fike, Jeff Glaubitz, Matt Hale, Cecilia Hennessy, Kevin Kazacos, Emily Latch, Nate Lichti, Kristen Page, Shilpi Sarkar, Pat Zollner and members of the Rhodes' lab for their help at various stages of this study. We thank the four anonymous reviewers and our associate editor for insightful and constructive comments on the previous versions of this paper. Funding for this research was provided by Purdue University.

\section{References}

Archie EA, Luikart G, Ezenwa VO (2009). Infecting epidemiology with genetics: a new frontier in disease ecology. Trends Ecol Evol 24: 21-30.

Beasley JC, Devault TL, Rhodes Jr OE (2007). Home-range attributes of raccoons in a fragmented agricultural region of northern Indiana. J Wildl Manage 71: 844-850.

Beasley JC, Rhodes Jr OE (2008). Relationship between raccoon abundance and crop damage. Human-Wildlife Conflicts 2: 248-259.

Belkhir K, Borsa P, Chikhi L, Raufaste N, Bonhomme F (2004). GENETIX 404, Windows software for population genetics. Laboratoire Génome, Populations, Interactions, CNRS UMR 5000, Université de Montpellier II, Montpellier, France (program available from: http://www.univ-montp2.fr/ genetix/genetix/genetix.htm).

Boone MD, Rhodes Jr OE (1996). Genetic structure among subpopulations of the eastern wild turkey (Meleagris gallopavo silvestris). Am Midl Nat 135: 168-171.

Burnham KP, Anderson DR (2002). Model Selection and Multimodel Inference: A Practical Information-Theoretic Approach, 2nd edn. Springer: New York.

Butler K, Field C, Herbinger CM, Smith BR (2004). Accuracy, efficiency and robustness of four algorithms allowing full sibship reconstruction from DNA marker data. Mol Ecol 13: 1589-1600.

Caillaud D, Prugnolle F, Durand P, Theron A, de Meeûs T (2006). Host sex and parasite genetic diversity. Microbes Infect 8: 2477-2483.

Chevillon C, Koffi BB, Barre N, Durand P, Arnathau C, de Meeûs T (2007). Direct and indirect inferences on parasite mating and gene transmission patterns-Pangamy in the cattle tick Rhipicephalus (Boophilus) microplus. Infect Genet Evol 7: 298-304.

Corander J, Marttinen P, Sirén J, Tang J (2008). Enhanced bayesian modelling in BAPS software for learning genetic structures of populations. BMC Bioinformatics 9: 539 .

Criscione CD (2008). Parasite co-structure: broad and local scale approaches. Parasite 15: 439-443.

Criscione CD, Blouin MS (2005). Effective sizes of macroparasite populations: a conceptual model. Trends Parasitol 21: 212-217.

Criscione CD, Poulin R, Blouin MS (2005). Mol Ecol of parasites: elucidating ecological and microevolutionary processes. Mol Ecol 14: 2247-2257.

Criscione CD, Anderson JD, Raby K, Sudimack D, Subedi J, Rai DR et al (2007). Microsatellite markers for the human nematode parasite Ascaris lumbricoides: development and assessment of utility. J Parasitol 93: 704-708.

Dakin EE, Avise JC (2004). Microsatellite null alleles in parentage analysis. Heredity 93: 504-509.

Darsie RF, Anastos G (1957). Geographical distribution and hosts of Ixodes texanus Banks (Acarina, Ixodidae). Ann Ent Soc Amer 50: 295-301.

de Meeûs T, Goudet J (2007). A step-by-step tutorial to use HierFstat to analyse populations hierarchically structured at multiple levels. Infect Genet Evol 7: 731-735.

de Meeûs T, Humair PF, Grunau C, Delaye C, Renaud F (2004). Non-Mendelian transmission of alleles at microsatellite loci: an example in Ixodes ricinus, the vector of Lyme disease. Int J Parasitol 34: 943-950.

de Meeûs T, McCoy KD, Prugnolle F, Chevillon C, Durand P, Hurtrez-Bousses S et al. (2007). Population genetics and molecular epidemiology or how to debusquer la bete. Infect Genet Evol 7: 308-332.

Dharmarajan G, Beasley JC, Fike JA, Rhodes Jr OE (2009a). Population genetic structure of raccoons (Procyon lotor) inhabiting a highly fragmented landscape. Can J Zool 87: 814-824.

Dharmarajan G, Beasley JC, Rhodes Jr OE (2010). Spatial and temporal factors affecting parasite genotypes encountered by 
hosts: empirical data from American dog ticks (Dermacentor variabilis) parasitising raccoons (Procyon lotor). Int J Parasitol 40: 787-795.

Dharmarajan G, Fike JA, Beasley JC, Rhodes Jr OE (2009b). Development and characterization of 14 polymorphic microsatellite loci in the raccoon tick (Ixodes texanus). Mol Ecol Res 9: 296-298.

Drauch AM, Fisher BE, Latch EK, Fike JA, Rhodes Jr OE (2008). Evaluation of a remnant lake sturgeon population's utility as a source for reintroductions in the Ohio River system. Conserv Genet 9: 1195-1209.

Goudet J (2005). HIERFSTAT, a package for R to compute and test hierarchical F-statistics. Mol Ecol Notes 5: 184-186.

Guzinski J, Bull CM, Donnellan SC, Gardner MG (2009). Molecular genetic data provide support for a model of transmission dynamics in an Australian reptile tick, Bothriocroton hydrosauri. Mol Ecol 18: 227-234.

Herbinger CM (2005). PEDIGREE. Available from http:// herbinger.biology.dal.ca:5080/Pedigree/.

Jongejan F, Uilenberg G (2004). The global importance of ticks. Parasitology 129: S3-S14.

Keeney DB, Waters JM, Poulin R (2007). Clonal diversity of the marine trematode Maritrema novaezealandensis within intermediate hosts: the Mol Ecol of parasite life cycles. Mol Ecol 16: 431-439.

Lafferty KD, Kuris AM (2002). Trophic strategies, animal diversity and body size. Trends Ecol Evol 17: 507-513.

Latch EK, Dharmarajan G, Glaubitz JC, Rhodes Jr OE (2006). Relative performance of Bayesian clustering software for inferring population substructure and individual assignment at low levels of population differentiation. Conserv Genet 7: 295-302.

Latch EK, Rhodes E (2006). Evidence for bias in estimates of local genetic structure due to sampling scheme. Anim Conserv 9: 308-315.

Lewis PO, Zaykin D (2001). Genetic Data Analysis: Computer program for the analysis of allelic data. Version 1.0 (d16c). Available from http://lewis.eeb.uconn.edu/lewishome/ software.html.

Luo HY, Nie P, Zhang YA, Yao WJ, Wang GT (2003). Genetic differentiation in populations of the cestode Bothriocephalus acheilognathi (Cestoda Pseudophyllidea) as revealed by eight microsatellite markers. Parasitology 126: 493-501.

McCoy KD (2008). The population genetic structure of vectors and our understanding of disease epidemiology. Parasite 15: 444-448.

McCoy KD, Tirard C, Michalakis Y (2003). Spatial genetic structure of the ectoparasite Ixodes uriae within breeding cliffs of its colonial seabird host. Heredity 91: 422-429.

Nei M (1987). Molecular Evolutionary Genetics. Columbia University Press: New York.

Picard D, Plantard O, Scurrah M, Mugniery D (2004). Inbreeding and population structure of the potato cyst nematode (Globodera pallida) in its native area (Peru). Mol Ecol 13: 2899-2908.

Plantard O, Picard D, Valette S, Scurrah M, Grenier E, Mugniery D (2008). Origin and genetic diversity of Western European populations of the potato cyst nematode (Globodera pallida) inferred from mitochondrial sequences and microsatellite loci. Mol Ecol 17: 2208-2218.

Plantard O, Porte C (2004). Population genetic structure of the sugar beet cyst nematode Heterodera schachtii: a gonochoristic and amphimictic species with highly inbred but weakly differentiated populations. Mol Ecol 13: 33-41.

Pritchard JK, Stephens M, Donnelly P (2000). Inference of population structure using multilocus genotype data. Genetics 155: 945-959.

Pudovkin AI, Zaykin DV, Hedgecock D (1996). On the potential for estimating the effective number of breeders from heterozygote-excess in progeny. Genetics 144: 383-387.

Ravel S, de Meeûs T, Dujardin JP, Zeze DG, Gooding RH, Dusfour I et al (2007). The tsetse fly Glossina palpalis palpalis is composed of several genetically differentiated small populations in the sleeping sickness focus of Bonon, Cote d'Ivoire. Infect Genet Evol 7: 116-125.

Roed KH, Hasle G, Midthjell V, Skretting G, Leinaas HP (2006). Identification and characterization of 17 microsatellite primers for the tick, Ixodes ricinus, using enriched genomic libraries. Mol Ecol Notes 6: 1165-1167.

Rosner B (2006). Fundamentals of Biostatistics 6th edn. Duxbury, Thomson-Brooks and Cole: Belmont, CA.

Rousset F (2008). GENEPOP ‘ 007: a complete re-implementation of the GENEPOP software for Windows and Linux. Mol Ecol Res 8: 103-106.

Smith BR, Herbinger CM, Merry HR (2001). Accurate partition of individuals into full-sib families from genetic data without parental information. Genetics 158: 1329-1338.

Sokal RR, Rohlf FJ (1981). Biometry: The Principles and Practice of Statistics in Biological Research 2nd edn. Freeman: New York.

Sonenshine DE (1993). Biology of Ticks vol. 2. Oxford University Press: New York.

Sugg DW, Chesser RK, Dobson FS, Hoogland JL (1996). Population genetics meets behavioral ecology. Trends Ecol Evol 11: 338-342.

Thiele EA, Sorensen RE, Gazzinelli A, Minchella DJ (2008). Genetic diversity and population structuring of Schistosoma mansoni in a Brazilian village. Int J Parasitol 38: 389-399.

Van Oosterhout C, Hutchinson WF, Wills DPM, Shipley P (2004). MICRO-CHECKER: software for identifying and correcting genotyping errors in microsatellite data. Mol Ecol Notes 4: 535-538.

Wagner AP, Creel S, Kalinowski ST (2006). Estimating relatedness and relationships using microsatellite loci with null alleles. Heredity 97: 336-345.

Wahlund S (1928). Zusammensetzung von Populationen und Korrelationserscheinungen von Standpunkt der Vererbungslehre aus betrachtet. Hereditas, 11, 65-106. In: Weiss KM and Ballanoff PA (eds). Demographic Genetics. Dowden, Hutchinson and Ross Inc.: Pennsylvania. pp 224-263.

Weir BS, Cockerham CC (1984). Estimating F statistics for the analysis of population structure. Evolution 38: 1358-1370.

Wielgoss S, Taraschewski H, Meyer A, Wirth T (2008). Population structure of the parasitic nematode Anguillicola crassus, an invader of declining North Atlantic eel stocks. Mol Ecol 17: 3478-3495.

Supplementary Information accompanies the paper on Heredity website (http://www.nature.com/hdy) 